\title{
ON PRINCIPAL CONGRUENCES IN DISTRIBUTIVE LATTICES WITH A COMMUTATIVE MONOIDAL OPERATION AND AN IMPLICATION
}

\author{
RAMON JANSANA AND HERNÁN JAVIER SAN MARTÍN
}

\begin{abstract}
In this paper we introduce and study a variety of algebras that properly includes integral distributive commutative residuated lattices and weak Heyting algebras. Our main goal is to give a characterization of the principal congruences in this variety. We apply this description in order to study compatible functions.
\end{abstract}

\section{INTRODUCTION}

It is very convenient to have good descriptions of the principal congruences of the algebras of a variety. One type of description is having first-order definable principal congruences. A much simpler and useful type of description is having equationally definable principal congruences. This concept was introduced in [20, 21]. Recall that a variety $\mathrm{V}$ has equationally definable principal congruences (EDPC) if there exists a finite family of quaternary terms $\left\{u_{i}, v_{i}\right\}_{i=1}^{r}$ such that for every algebra $A$ in $\mathrm{V}$ and every principal congruence $\theta(a, b)$ of $A$, it holds that $(c, d) \in \theta(a, b)$ if and only if $u_{i}(a, b, c, d)=v_{i}(a, b, c, d)$ for each $i=1, \ldots, r$. The property EDPC is also of logical interest because an algebraizable logic whose equivalent algebraic semantics is a variety $\mathrm{V}$ has some form of deduction-detachment theorem if and only if the variety $\mathrm{V}$ has EDPC, a consequence of a more general result proved by Blok and Pigozzi in [5, Thm. 5.5](see also [19, Thm. 3.85]).

There are varieties that do not have EDPC but where it is still possible to have a good characterization of the principal congruences with the following local version of EDPC: there exists a finite family of quaternary terms $\left\{u_{(i, n, k)}, v_{(i, n, k)}\right\}_{i=1}^{r}$ (with $n, k \geq 0)$ such that for every principal congruence $\theta(a, b)$ of any algebra $A$ in the variety it holds that $(c, d) \in \theta(a, b)$ if and only if there exist $n, k \geq 0$ such that $u_{(i, n, k)}(a, b, c, d)=v_{(i, n, k)}(a, b, c, d)$ for every $i=1, \ldots r$. We say that a variety of algebras has locally equationally definable principal congruences if there exists a finite family of quaternary terms such that in every algebra of the variety they define the principal congruences in the way just described. In particular, the variety of commutative residuated lattices has locally equationally definable principal congruences (it can be deduced from results of 11) and the variety of weak Heyting algebras has this property too [29, Theorem 2.2]. The concept of locally equationally definable principal congruences for quasivarieties is introduced in 4, in an equivalent form. From the logical point of view it is related to the notion of having a local deduction theorem.

The main goal of the paper is to prove that a variety of algebras that properly includes the integral distributive commutative residuated lattices 23 and the weak Heyting algebras [14, 3] has locally equationally definable principal congruences. We

\footnotetext{
${ }^{1}$ If $A$ is an algebra and $a, b \in A$, then $\theta(a, b)$ denotes the principal congruence of $A$ generated by $(a, b)$, i.e., the smallest congruence of $A$ that contains $(a, b)$.
} 
call the members of this variety distributive lattices with a commutative monoidal operation and an implication and we denote the variety by DLCMI.

The second goal of the paper is the study of compatible functions of algebras in DLCMI, by applying the characterization of the principal congruences. Given an algebra $A$ and a function $f: A^{n} \rightarrow A$, we say that $f$ is compatible if every congruence of $A$ is a congruence of $A$ enriched with $f$ as a new operation. The principal congruences are closely related to compatible functions. For instance, if $f: A \rightarrow A$ is a function, then $f$ is compatible if and only if $(f(a), f(b)) \in \theta(a, b)$ for every $a, b \in A$. Furthermore, certain algebraizable logics whose equivalent algebraic semantics are varieties are also connected with compatible functions. Caicedo showed in [8] that in any axiomatic expansion $\mathrm{L}^{\prime}$ of an algebraizable logic $\mathrm{L}$ by adding only new connectives that are implicitly definable, the new connectives can be translated to compatible functions (whenever they exist) in the algebras of the equivalent algebraic semantics of the initial logic L. Therefore, the principal congruences in a variety that is the equivalent algebraic semantics of an algebraizable logic L (which has an axiomatic expansion $\mathrm{L}^{\prime}$ with the above mentioned property) is also strongly linked with properties of $\mathrm{L}^{\prime}$.

The paper is organized as follows. In Section 2 we provide the basic definitions and results. In Section 3 we show that DLCMI has locally equationally definable principal congruences. In particular, we obtain known characterizations of the principal congruences of weak Heyting algebras and integral distributive commutative residuated lattices. The first one was obtained in [29], and the second is part of the folklore and it follows easily from [1. In Section 4 we apply the results of the previous section to study compatible functions in DLCMI. Finally, in Section 5 we establish other connections with existing literature.

\section{BASIC DEFINITIONS AND RESUlTS}

In what follows we start by recalling the definitions of commutative residuated lattice and weak Heyting algebra respectively.

Definition 1. An algebra $(A, \wedge, \vee, \cdot, \rightarrow, e)$ of type $(2,2,2,2,0)$ is said to be a commutative residuated lattice if the following conditions are satisfied:

(1) $(A, \cdot, e)$ is a commutative monoid,

(2) $(A, \wedge, \vee)$ is a lattice,

(3) for every $a, b, c \in A, a \cdot b \leq c$ if and only if $a \leq b \rightarrow c$.

A commutative residuated lattice $(A, \wedge, \vee, \cdot, \rightarrow, e)$ is distributive if its lattice reduct is distributive and it is integral if the unit of the monoid is the largest element of the lattice reduct. We write IDCRL for the variety of integral distributive commutative residuated lattices. Since the class of commutative residuated lattices is a variety, then IDCRL is a variety.

Definition 2. An algebra $(A, \wedge, \vee, \rightarrow, 0,1)$ of type $(2,2,2,0,0)$ is a weak Heyting algebra if the reduct algebra $(A, \wedge, \vee, 0,1)$ is a bounded distributive lattice and $\rightarrow$ is a binary operation such that satisfies the following conditions for all $a, b, c \in A$ :

(1) $(a \rightarrow b) \wedge(a \rightarrow c)=a \rightarrow(b \wedge c)$,

(2) $(a \rightarrow c) \wedge(b \rightarrow c)=(a \vee b) \rightarrow c$,

(3) $(a \rightarrow b) \wedge(b \rightarrow c) \leq a \rightarrow c$

(4) $a \rightarrow a=1$.

We denote by $\mathrm{WH}$ the variety of weak Heyting algebras.

In what follows we introduce a variety that properly contains the variety of integral distributive commutative residuated lattices and the variety of weak Heyting algebras. This is the variety that we study in this paper. 
Definition 3. An algebra $(A, \wedge, \vee, \cdot, \rightarrow, 1)$ of type $(2,2,2,2,0)$ is a distributive lattice with a commutative monoidal operation and an implication if for every $a, b, c \in A$ the following conditions are satisfied:

(1) $(A, \wedge, \vee)$ is a distributive lattice,

(2) 1 is the largest element of $(A, \wedge, \vee)$,

(3) $(A, \cdot, 1)$ is a commutative monoid,

(4) $(a \rightarrow b) \wedge(a \rightarrow c)=a \rightarrow(b \wedge c)$,

(5) $(a \rightarrow c) \wedge(b \rightarrow c)=(a \vee b) \rightarrow c$,

(6) $a \rightarrow a=1$,

(7) $(a \vee b) \cdot c=(a \cdot c) \vee(b \cdot c)$,

(8) $(a \rightarrow b) \cdot(b \rightarrow c) \leq a \rightarrow c$,

(9) $a \rightarrow b \leq(a \cdot c) \rightarrow(b \cdot c)$.

We denote by DLCMI the variety of algebras given in Definition 3 and we refer to its members as DLCMIs.

Let $A \in \mathrm{DLCMI}$. The following conditions are satisfied for all $a, b, c \in A$ :

1) If $a \leq b$, then $a \cdot c \leq b \cdot c, b \rightarrow c \leq a \rightarrow c$, and $c \rightarrow a \leq c \rightarrow b$.

2) $a \cdot b \leq a \wedge b$.

3) $a \cdot b \leq a$.

4) $1 \rightarrow a \leq b \rightarrow(a \cdot b)$.

Lemma 1. IDCRL is a subvariety of DLCMI.

Proof. It follows from straightforward computations based on properties of integral commutative residuated lattices [23].

Lemma 2. The variety $\mathrm{WH}$ can be seen as a subvariety of DLCMI, namely the subvariety that is defined by the equation $x \wedge y \approx x \cdot y$. More precisely, we have that $(A, \wedge, \vee, \rightarrow, 0,1) \in \mathrm{WH}$ if and only if $(A, \wedge, \vee, \wedge, \rightarrow, 1) \in \mathrm{DLCMI}$.

Proof. In order to show that WH can be seen as a subvariety of DLCMI, we will prove that the inequality $a \rightarrow b \leq(a \wedge c) \rightarrow(b \wedge c)$ holds in weak Heyting algebras, since the rest of the items can be proved easily. Since $a \wedge c \leq a$, then $a \rightarrow b \leq(a \wedge c) \rightarrow b$. But $(a \wedge c) \rightarrow(b \wedge c)=(a \wedge c) \rightarrow b$, so we conclude that $a \rightarrow b \leq(a \wedge c) \rightarrow(b \wedge c)$.

Now we will give an example of an algebra in DLCMI that is neither a weak Heyting algebra nor an integral distributive commutative residuated lattice.

Example 1. Let $H_{3}=\{0, a, 1\}$ be the chain of three elements with $0<a<1$. The algebra $\left(H_{3}, \wedge, \vee, \rightarrow, 1\right)$ belongs to $\mathrm{WH}$, where $\rightarrow$ is given by $x \rightarrow y=1$ for every $x, y \in H_{3}$. Following the convention given in Lemma 2 we also can say that $H_{3}^{\mathrm{WH}}=\left(H_{3}, \wedge, \vee, \wedge, \rightarrow, 1\right) \in \mathrm{WH}$. Let $H_{3}^{\mathrm{MV}}=\left(H_{3}, \wedge, \vee, \odot, \rightarrow, 1\right)$ be the MV-chain of three elements presented as a residuated lattice [16. The product and implication $\rightarrow$ in $H_{3}^{\mathrm{MV}}$ are given in the following tables:

\begin{tabular}{|c|c|c|c|c|c|c|c|}
\hline$\odot$ & 0 & $a$ & 1 & $\rightarrow$ & 0 & $a$ & 1 \\
\hline 0 & 0 & 0 & 0 & 0 & 1 & 1 & 1 \\
\hline$a$ & 0 & 0 & $a$ & $a$ & $a$ & 1 & \\
\hline 1 & 0 & $a$ & 1 & 1 & 0 & $a$ & 1 \\
\hline
\end{tabular}

We define $A$ as the product algebra $H_{3}^{\mathrm{MV}} \times H_{3}^{\mathrm{WH}}$. Since $H_{3}^{\mathrm{MV}}, H_{3}^{\mathrm{WH}} \in \mathrm{DLCMI}$ and DLCMI is a variety, then $A \in \operatorname{DLCMI}$. Since $(a, a) \cdot(a, a)=(0, a),(a, a) \wedge$ $(a, a)=(a, a)$ and $0 \neq a$, then $(a, a) \cdot(a, a) \neq(a, a) \wedge(a, a)$, so $A \notin \mathrm{WH}$. In order to show that $A \notin \mathrm{IDCRL}$ notice that $(x, y) \cdot(z, w)=(x \odot z, y \wedge w)$ for every $x, y, z, w \in H_{3}$. Since $(a, a) \rightarrow(0,0)=(a, 1)$ then $(a, a) \leq(a, a) \rightarrow(0,0)$. However $(a, a) \cdot(a, a)=(0, a) \not \leq(0,0)$. Therefore, $A \notin \operatorname{IDCRL}$. 
It follows from Example1 that WH and IDCRL are proper subvarieties of DLCMI. Besides, WH and IDCRL are incomparable varieties. In order to show it, first note that $H_{3}^{\mathrm{MV}} \in \mathrm{IDCRL}$ and $H_{3}^{\mathrm{MV}} \notin \mathrm{WH}$ because $a \odot a=0$ and $a \wedge a \neq 0$. Finally note that $H_{3}^{\mathrm{WH}} \in \mathrm{WH}$ and $H_{3}^{\mathrm{WH}} \notin \mathrm{IDCRL}$, which follows from the fact that $1 \leq 1=1 \rightarrow a$ and $1 \wedge 1=1 \not \leq a$.

We have the following picture:

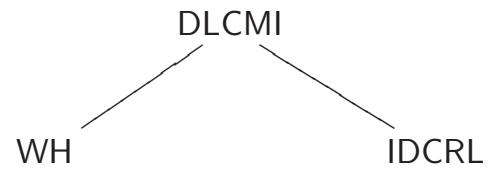

The following elementary lemma allows us to give an alternative presentation for DLCMIs.

Lemma 3. Let $A$ be an algebra of type $(2,2,2,2,0)$ which satisfies the conditions 1),..,8) of Definition 目. The following conditions are equivalent:

1) A satisfies condition 9) of Definition 目.

2) For every $a, b, c, d \in A,(a \rightarrow b) \cdot(c \rightarrow d) \leq(a \cdot c) \rightarrow(b \cdot d)$.

Proof. Assume condition 9). We have that $a \rightarrow b \leq(a \cdot c) \rightarrow(b \cdot c)$ and $c \rightarrow d \leq$ $(b \cdot c) \rightarrow(b \cdot d)$. Then

$$
\begin{aligned}
(a \rightarrow b) \cdot(c \rightarrow d) & \leq((a \cdot c) \rightarrow(b \cdot c)) \cdot((b \cdot c) \rightarrow(b \cdot d)) \\
& \leq(a \cdot c) \rightarrow(b \cdot d) .
\end{aligned}
$$

Conversely, suppose that for every $a, b, c, d \in A$ the inequality $(a \rightarrow b) \cdot(c \rightarrow d) \leq$ $(a \cdot c) \rightarrow(b \cdot d)$ is satisfied. Then

$$
\begin{aligned}
a \rightarrow b & =(a \rightarrow b) \cdot 1 \\
& =(a \rightarrow b) \cdot(c \rightarrow c) \\
& \leq(a \cdot c) \rightarrow(b \cdot c),
\end{aligned}
$$

which was our aim.

\section{Principal CONGRUEnCES}

In 14 it was proved that WH does not have EDPC. Hence, DLCMI does not have EDPC either. In this section we prove that DLCMI has locally equationally definable principal congruences.

Definition 4. A variety of algebras $\mathrm{V}$ has locally equationally definable principal congruences if there exists a finite family of quaternary terms $\left\{u_{(i, n, k)}, v_{(i, n, k)}\right\}_{i=1}^{r}$ (with $n, k \geq 0$ ) such that for every $A \in \mathrm{V}$ and $a, b \in A$ it holds that for every $c, d \in$ $A,(c, d) \in \theta(a, b)$ if and only if there exist $n, k \geq 0$ such that $u_{(i, n, k)}(a, b, c, d)=$ $v_{(i, n, k)}(a, b, c, d)$ for every $i=1, \ldots r$.

We start with some preliminary definitions and technical results.

If $A$ is an algebra, we denote by $\operatorname{Con}(A)$ the lattice of the congruences of $A$. We refer by $\mathrm{DL}$ to the variety of distributive lattices [2, 6]. The following lemma is part of the folklore of distributive lattices.

Lemma 4. Let $A \in \mathrm{DL}, \theta \in \operatorname{Con}(A)$ and $a, b \in A$. If there is $c \in A$ such that $(a \wedge c, b \wedge c) \in \theta$ and $(a \vee c, b \vee c) \in \theta$, then $(a, b) \in \theta$.

Lemma 5. Let $A \in \mathrm{DL}, \theta \in \operatorname{Con}(A),(a, b) \in \theta$ and $c, d \in A$.

$$
\text { If }(c \wedge a \wedge b, d \wedge a \wedge b),(c \vee a \vee b, d \vee a \vee b) \in \theta \text {, then }(c, d) \in \theta \text {. }
$$


Proof. Since $(a, b) \in \theta$, then $(a \wedge b, b) \in \theta$ and $(a \vee b, b) \in \theta$. Thus, $(a \wedge b, a \vee b) \in \theta$. This implies that $(c \vee(a \wedge b), c \vee a \vee b) \in \theta$ and $(d \vee(a \wedge b), d \vee a \vee b) \in \theta$. Since by hypothesis $(c \vee a \vee b, d \vee a \vee b) \in \theta$ then

$$
(c \vee(a \wedge b), d \vee(a \wedge b)) \in \theta
$$

By hypothesis we also have that

$$
(c \wedge(a \wedge b), d \wedge(a \wedge b)) \in \theta
$$

Therefore, it follows from (11), (2) and Lemma 4 that $(c, d) \in \theta$.

Let $A \in \mathrm{DLCMI}, n \geq 1$ and $a, b \in A$. We define inductively $a^{n}$ by setting $a^{0}:=1$ and $a^{n}:=a \cdot a^{n-1}$. We also define $\square^{0}(a)=a, \square(a)=1 \rightarrow a$ and the iterated operation $\square^{n}$ in the usual way. We also define

$$
\begin{gathered}
a \leftrightarrow b:=(a \rightarrow b) \wedge(b \rightarrow a), \\
t_{n}(a, b):=\square^{0}(a \leftrightarrow b) \wedge \square(a \leftrightarrow b) \wedge \cdots \wedge \square^{n}(a \leftrightarrow b) .
\end{gathered}
$$

Remark 1. Let $A \in \mathrm{DLCMI}, n \geq 1$ and $a, b \in A$.

a) If $k$ is a natural number, we write $t_{n}^{k}(a, b)$ in place of $\left(t_{n}(a, b)\right)^{k}$.

b) The map $\square$ preserves finite meets. In particular $\square$ is monotonic, i.e., if $a \leq b$ then $\square(a) \leq \square(b)$.

We will prove in Theorem 10 that DLCMI has locally equationally definable principal congruences by the set QT of the following quaternary terms:

- $u_{1, n, k}\left(x_{1}, x_{2}, y_{1}, y_{2}\right):=\left(t_{n}^{k}\left(x_{1}, x_{2}\right) \cdot\left(y_{1} \wedge x_{1} \wedge x_{2}\right)\right) \vee\left(y_{2} \wedge x_{1} \wedge x_{2}\right)$,

- $v_{1, n, k}\left(x_{1}, x_{2}, y_{1}, y_{2}\right):=y_{2} \wedge x_{1} \wedge x_{2}$,

- $u_{2, n, k}\left(x_{1}, x_{2}, y_{1}, y_{2}\right):=\left(t_{n}^{k}\left(x_{1}, x_{2}\right) \cdot\left(y_{2} \wedge x_{1} \wedge x_{2}\right)\right) \vee\left(y_{1} \wedge x_{1} \wedge x_{2}\right)$,

- $v_{2, n, k}\left(x_{1}, x_{2}, y_{1}, y_{2}\right):=y_{1} \wedge x_{1} \wedge x_{2}$,

- $u_{3, n, k}\left(x_{1}, x_{2}, y_{1}, y_{2}\right):=\left(t_{n}^{k}\left(x_{1}, x_{2}\right) \cdot\left(y_{1} \vee x_{1} \vee x_{2}\right)\right) \vee\left(y_{2} \vee x_{1} \vee x_{2}\right)$,

- $v_{3, n, k}\left(x_{1}, x_{2}, y_{1}, y_{2}\right):=y_{2} \vee x_{1} \vee x_{2}$,

- $u_{4, n, k}\left(x_{1}, x_{2}, y_{1}, y_{2}\right):=\left(t_{n}^{k}\left(x_{1}, x_{2}\right) \cdot\left(y_{2} \vee x_{1} \vee x_{2}\right)\right) \vee\left(y_{1} \vee x_{1} \vee x_{2}\right)$,

- $v_{4, n, k}\left(x_{1}, x_{2}, y_{1}, y_{2}\right):=y_{1} \vee x_{1} \vee x_{2}$,

- $u_{5, n, k}\left(x_{1}, x_{2}, y_{1}, y_{2}\right):=t_{n}^{k}\left(x_{1}, x_{2}\right) \vee\left(y_{1} \leftrightarrow y_{2}\right)$,

- $v_{5, n, k}\left(x_{1}, x_{2}, y_{1}, y_{2}\right):=y_{1} \leftrightarrow y_{2}$.

with $n, k$ natural numbers.

To achieve our goal, for any $A \in \mathrm{DLCMI}$ and $a, b \in A$ we define the binary relation $R(a, b)$ as follows: $(c, d) \in R(a, b)$ if and only if there are natural numbers $n$ and $k$ that satisfy the following conditions:

(C1) $t_{n}^{k}(a, b) \cdot(c \wedge a \wedge b) \leq d \wedge a \wedge b$ and $t_{n}^{k}(a, b) \cdot(d \wedge a \wedge b) \leq c \wedge a \wedge b$,

(C2) $t_{n}^{k}(a, b) \cdot(c \vee a \vee b) \leq d \vee a \vee b$ and $t_{n}^{k}(a, b) \cdot(d \vee a \vee b) \leq c \vee a \vee b$,

(C3) $t_{n}^{k}(a, b) \leq c \leftrightarrow d$.

We say that $(n, k)$ is a pair of natural numbers associated with $(c, d)$.

Remark 2. Let $A \in \mathrm{DLCMI}$ and $a, b \in A$.

a) $t_{n+1}(a, b) \leq t_{n}(a, b)$ and $t_{n}^{k+1}(a, b) \leq t_{n}^{k}(a, b)$. Hence, $t_{m}^{p}(a, b) \leq t_{n}^{q}(a, b)$ whenever $n \leq m$ and $q \leq p$.

b) Let $(c, d),\left(c^{\prime}, d^{\prime}\right) \in R(a, b)$ and $(p, q),(r, s)$ pairs of natural numbers associated with $(c, d)$ and $\left(c^{\prime}, d^{\prime}\right)$ respectively. Consider $n:=\max \{p, r\}$ and $k:=\max \{q, s\}$. It follows from the previous item that $(n, k)$ is a pair of natural numbers associated with $(c, d)$ and $\left(c^{\prime}, d^{\prime}\right)$.

Let $A \in \mathrm{DL}$ and $\theta$ an equivalence relation on $A$. It is easily seen that in this case $\theta$ is a congruence if and only if the following two conditions hold: 
1) For every $a, b, c \in A$, if $(a, b) \in \theta$, then $(a \wedge c, b \wedge c) \in \theta$.

2) For every $a, b, c \in A$, if $(a, b) \in \theta$, then $(a \vee c, b \vee c) \in \theta$.

In the next lemma we will use the above mentioned property.

Lemma 6. Let $A \in \mathrm{DLCMI}$ and $a, b \in A$. Then $(a, b) \in R(a, b)$ and $R(a, b)$ is $a$ congruence of the lattice reduct of $A$.

Proof. First we prove that $(a, b) \in R(a, b)$. Note that since $a \leftrightarrow b \leq 1$, then $(a \leftrightarrow b) \cdot(a \wedge b) \leq a \wedge b$ and $(a \leftrightarrow b) \cdot(a \vee b) \leq a \vee b$. Besides $a \leftrightarrow b \leq a \leftrightarrow b$. Thus we have that conditions $(\mathrm{C} 1),(\mathrm{C} 2)$ and $(\mathrm{C} 3)$ in the definition of $R(a, b)$ hold for $(a, b)$. Therefore, $(a, b) \in R(a, b)$. Now we prove that $R(a, b)$ is a congruence of the lattice reduct of $A$.

(i) We will prove that $R(a, b)$ is an equivalence relation. The reflexivity and the symmetry are immediate. In order to prove the transitivity, consider $(c, d) \in R(a, b)$ and $(d, e) \in R(a, b)$. It follows from Remark 2 that there is a pair of natural numbers $(n, k)$ associated with $(c, d)$ and $(d, e)$. Then

$$
\begin{aligned}
t_{n}^{2 k}(a, b) \cdot(c \wedge a \wedge b) & =t_{n}^{k}(a, b) \cdot t_{n}^{k}(a, b) \cdot(c \wedge a \wedge b) \\
& \leq t_{n}^{k}(a, b) \cdot(d \wedge a \wedge b) \\
& \leq e \wedge a \wedge b .
\end{aligned}
$$

In a similar way it can be proved that $t_{n}^{2 k}(a, b) \cdot(e \wedge a \wedge b) \leq c \wedge a \wedge b, t_{n}^{2 k}(a, b) \cdot(c \vee$ $a \vee b) \leq e \vee a \vee b$, and $t_{n}^{2 k}(a, b) \cdot(e \vee a \vee b) \leq c \vee a \vee b$. Finally we will see that $t_{n}^{2 k}(a, b) \leq c \leftrightarrow e$. Note that $t_{n}^{k}(a, b) \leq c \rightarrow d, t_{n}^{k}(a, b) \leq d \rightarrow c, t_{n}^{k}(a, b) \leq d \rightarrow e$ and $t_{n}^{k}(a, b) \leq e \rightarrow d$. Then

$$
\begin{aligned}
t_{n}^{2 k}(a, b) & \leq(c \rightarrow d) \cdot(d \rightarrow e) \\
& \leq c \rightarrow e .
\end{aligned}
$$

In a similar way we can show that $t_{n}^{2 k}(a, b) \leq e \rightarrow c$. Thus,

$$
t_{n}^{2 k}(a, b) \leq(c \rightarrow e) \wedge(e \rightarrow c) .
$$

Hence, $R(a, b)$ is a transitive relation.

(ii) Let $(c, d) \in R(a, b)$. We will prove that $(c \wedge e, d \wedge e) \in R(a, b)$. We have that

$$
\begin{aligned}
t_{n}^{k}(a, b) \cdot((c \wedge e) \wedge a \wedge b) & \leq t_{n}^{k}(a, b) \cdot(c \wedge a \wedge b) \\
& \leq d \wedge a \wedge b
\end{aligned}
$$

and $t_{n}^{k}(a, b) \cdot((c \wedge e) \wedge a \wedge b) \leq e$. Thus, $t_{n}^{k}(a, b) \cdot((c \wedge e) \wedge a \wedge b) \leq(d \wedge e) \wedge a \wedge b$. The same argument shows that $t_{n}^{k}(a, b) \cdot((d \wedge e) \wedge a \wedge b) \leq(c \wedge e) \wedge a \wedge b$.

Now we will prove the inequality $t_{n}^{k}(a, b) \cdot((c \wedge e) \vee a \vee b) \leq(d \wedge e) \vee a \vee b$. First note that

$$
\begin{aligned}
t_{n}^{k}(a, b) \cdot((c \wedge e) \vee a \vee b) & \leq t_{n}^{k}(a, b) \cdot(c \vee a \vee b) \\
& \leq d \vee a \vee b .
\end{aligned}
$$

We also have that

$$
t_{n}^{k}(a, b) \cdot((c \wedge e) \vee a \vee b) \leq e \vee a \vee b .
$$

Then,

$$
t_{n}^{k}(a, b) \cdot((c \wedge e) \vee a \vee b) \leq(d \vee a \vee b) \wedge(e \vee a \vee b)
$$

Taking into account the distributivity of the underlying lattice of $A$ we obtain

$$
(d \vee a \vee b) \wedge(e \vee a \vee b)=(d \wedge e) \vee a \vee b .
$$

Hence, $t_{n}^{k}(a, b) \cdot((c \wedge e) \vee a \vee b) \leq(d \wedge e) \vee a \vee b$. We also have that

$$
t_{n}^{k}(a, b) \cdot((d \wedge e) \vee a \vee b) \leq(c \wedge e) \vee a \vee b .
$$

In what follows we will prove the inequality $t_{n}^{k}(a, b) \leq(c \wedge e) \leftrightarrow(d \wedge e)$. It is enough to prove the inequality $c \leftrightarrow d \leq(c \wedge e) \leftrightarrow(d \wedge e)$. First note that $(c \wedge e) \rightarrow(d \wedge e)=$ 
$((c \wedge e) \rightarrow d) \wedge((c \wedge e) \rightarrow e)$. Since $c \wedge e \leq c$, then $(c \wedge e) \rightarrow d \geq c \rightarrow d$ and since $c \wedge e \leq e$, then $(c \wedge e) \rightarrow e \geq e \rightarrow e=1$. Hence,

$$
\begin{aligned}
(c \wedge e) \rightarrow(d \wedge e) & =((c \wedge e) \rightarrow d) \wedge((d \wedge e) \rightarrow e) \\
& \geq c \rightarrow d .
\end{aligned}
$$

Analogously we obtain that $(d \wedge e) \rightarrow(c \wedge e) \geq d \rightarrow c$. Thus $c \leftrightarrow d \leq(c \wedge e) \leftrightarrow(d \wedge e)$. Thus, we have proved that $(c \wedge e, d \wedge e) \in R(a, b)$.

(iii) The fact $(c \vee e, d \vee e) \in R(a, b)$ whenever $(c, d) \in R(a, b)$ can be proved using similar ideas to the ones employed for the case (ii). Now in addition we use the property $(c \vee d) \cdot e=(c \cdot e) \vee(d \cdot e)$ for every $c, d, e \in A$.

The following lemma will play a fundamental role.

Lemma 7. Let $A \in \mathrm{DLCMI}$ and $a \in A$. Then $(1 \rightarrow a)^{n} \leq 1 \rightarrow a^{n}$ for every $n$.

Proof. Notice that for every $n$ the we have that

$$
\left(1 \rightarrow a^{n}\right) \cdot(1 \rightarrow a) \leq(1 \cdot 1) \rightarrow\left(a \cdot a^{n}\right),
$$

i.e.,

$$
\left(1 \rightarrow a^{n}\right) \cdot(1 \rightarrow a) \leq 1 \rightarrow a^{n+1} .
$$

Also notice that $(1 \rightarrow a)^{0} \leq 1 \rightarrow a^{0}$ and $(1 \rightarrow a)^{1} \leq 1 \rightarrow a^{1}$. Assume that $(1 \rightarrow a)^{n} \leq 1 \rightarrow a^{n}$ for some $n$. Taking into account (3) we prove the inequality $(1 \rightarrow a)^{n+1} \leq 1 \rightarrow a^{n+1}$ as follows:

$$
\begin{aligned}
(1 \rightarrow a)^{n+1} & =(1 \rightarrow a)^{n} \cdot(1 \rightarrow a) \\
\leq & \left(1 \rightarrow a^{n}\right) \cdot(1 \rightarrow a) \\
\leq & 1 \rightarrow a^{n+1} .
\end{aligned}
$$

Let $A \in \mathrm{DLCMI}$ and $a, b \in A$. Let $(c, d),(u, w) \in R(a, b)$. It follows from Remark 2 that there exists a pair of natural numbers $(n, k)$ associated with $(c, d)$ and $(u, w)$. In particular, $(n, 2 k)$ is also a pair of natural numbers associated with $(c, d)$ and $(u, w)$. The pair $(n, 2 k)$ will be considered in the following two lemmas.

Lemma 8. Let $A \in \mathrm{DLCMI}$ and $a, b \in A$. Let $(c, d),(u, w) \in R(a, b)$. There are natural numbers $n$ and $k$ that satisfy the following conditions:

1) $t_{n}^{2 k}(a, b) \cdot(c \rightarrow u) \leq d \rightarrow w$,

2) $t_{n}^{2 k}(a, b) \cdot((c \rightarrow u) \wedge a \wedge b) \leq(d \rightarrow w) \wedge a \wedge b$,

3) $t_{n}^{2 k}(a, b) \cdot((d \rightarrow w) \wedge a \wedge b) \leq(c \rightarrow u) \wedge a \wedge b$,

4) $t_{n}^{2 k}(a, b) \cdot((c \rightarrow u) \vee a \vee b) \leq(d \rightarrow w) \vee a \vee b$,

5) $t_{n}^{2 k}(a, b) \cdot((d \rightarrow w) \vee a \vee b) \leq(c \rightarrow u) \vee a \vee b$,

6) $t_{n+1}^{2 k}(a, b) \leq(c \rightarrow u) \leftrightarrow(d \rightarrow w)$.

Proof. First note that $t_{n}^{k}(a, b) \leq c \rightarrow d, d \rightarrow c, u \rightarrow w, w \rightarrow u$. Then

$$
\begin{aligned}
t_{n}^{2 k}(a, b) \cdot(c \rightarrow u) & =t_{n}^{k}(a, b) \cdot t_{n}^{k}(a, b) \cdot(c \rightarrow u) \\
\leq & (d \rightarrow c) \cdot(u \rightarrow w) \cdot(c \rightarrow u) \\
& =(d \rightarrow c) \cdot(c \rightarrow u) \cdot(u \rightarrow w) \\
& \leq d \rightarrow w .
\end{aligned}
$$

Thus,

$$
t_{n}^{2 k}(a, b) \cdot(c \rightarrow u) \leq d \rightarrow w,
$$

which is condition 1 ).

We will prove that condition 1) implies condition 2). First note that

$$
\begin{aligned}
t_{n}^{2 k}(a, b) \cdot((c \rightarrow u) \wedge a \wedge b) & \leq t_{n}^{2 k}(a, b) \cdot(c \rightarrow u) \\
& \leq d \rightarrow w .
\end{aligned}
$$


Besides,

$$
t_{n}^{2 k}(a, b) \cdot((c \rightarrow u) \wedge a \wedge b) \leq a \wedge b
$$

Then $t_{n}^{2 k}(a, b) \cdot((c \rightarrow u) \wedge a \wedge b) \leq(d \rightarrow w) \wedge a \wedge b$, which is condition 2). Similarly it can be proved that

$$
t_{n}^{2 k}(a, b) \cdot((d \rightarrow w) \wedge a \wedge b) \leq(c \rightarrow u) \wedge a \wedge b,
$$

which is condition 3 ).

Now we will prove the condition 4). It follows from (4) that

$$
\begin{aligned}
t_{n}^{2 k}(a, b) \cdot((c \rightarrow u) \vee a \vee b) & \left.=\left(t_{n}^{2 k}(a, b) \cdot(c \rightarrow u)\right) \vee\left(t_{n}^{2 k}(a, b)\right) \cdot(a \vee b)\right) \\
\leq & (d \rightarrow w) \vee a \vee b,
\end{aligned}
$$

which is condition 4). Analogously we can show that

$$
t_{n}^{2 k}(a, b) \cdot((d \rightarrow w) \vee a \vee b) \leq(c \rightarrow u) \vee a \vee b,
$$

i.e., condition 5).

Finally we will prove condition 6). It follows from display (4) that

$$
(c \rightarrow u) \rightarrow\left((c \rightarrow u) \cdot t_{n}^{2 k}(a, b)\right) \leq(c \rightarrow u) \rightarrow(d \rightarrow w) .
$$

Taking into account the inequality $1 \rightarrow c_{1} \leq c_{2} \rightarrow\left(c_{1} \cdot c_{2}\right)$ with $c_{1}=t_{n}^{2 k}(a, b)$ and $c_{2}=c \rightarrow u$ we obtain that

$$
1 \rightarrow t_{n}^{2 k}(a, b) \leq(c \rightarrow u) \rightarrow\left((c \rightarrow u) \cdot t_{n}^{2 k}(a, b)\right) .
$$

Besides, it follows from Lemma 7 that

$$
\left(1 \rightarrow t_{n}(a, b)\right)^{2 k} \leq 1 \rightarrow t_{n}^{2 k}(a, b) .
$$

Since $t_{n+1}(a, b) \leq \square(a \leftrightarrow b) \wedge \cdots \wedge \square^{n+1}(a \leftrightarrow b)$ and

$$
\square(a \leftrightarrow b) \wedge \cdots \wedge \square^{n+1}(a \leftrightarrow b)=1 \rightarrow t_{n}(a, b),
$$

then $t_{n+1}(a, b) \leq 1 \rightarrow t_{n}(a, b)$, so $t_{n+1}^{2 k}(a, b) \leq\left(1 \rightarrow t_{n}(a, b)\right)^{2 k}$. Thus, it follows from (5), (6) and (7) that $t_{n+1}^{2 k}(a, b) \leq(c \rightarrow u) \rightarrow(d \rightarrow w)$. Similarly we can show the inequality $t_{n+1}^{2 k}(a, b) \leq(d \rightarrow w) \rightarrow(c \rightarrow u)$. Therefore,

$$
t_{n+1}^{2 k}(a, b) \leq(c \rightarrow u) \leftrightarrow(d \rightarrow w) .
$$

Lemma 9. Let $A \in \mathrm{DLCMI}$ and $a, b \in A$. Let $(c, d) \in R(a, b)$ and $(u, w) \in R(a, b)$. There exist natural numbers $n$ and $k$ that satisfy the following conditions:
1) $t_{n}^{2 k}(a, b)((c \cdot u) \wedge a \wedge b) \leq(d \cdot w) \wedge a \wedge b$,
2) $t_{n}^{2 k}(a, b)((d \cdot w) \wedge a \wedge b) \leq(c \cdot u) \wedge a \wedge b$
3) $t_{n}^{2 k}(a, b)((c \cdot u) \vee a \vee b) \leq(d \cdot w) \vee a \vee b$,
4) $t_{n}^{2 k}(a, b)((d \cdot w) \vee a \vee b) \leq(c \cdot u) \vee a \vee b$,
5) $t_{n}^{2 k}(a, b) \leq(c \cdot u) \leftrightarrow(d \cdot w)$.

Proof. First note that

$$
\begin{aligned}
t_{n}^{k}(a, b) \cdot((c \cdot u) \wedge a \wedge b) & \leq t_{n}^{k}(a, b) \cdot(c \wedge a \wedge b) \\
& \leq d \wedge a \wedge b \\
& \leq d
\end{aligned}
$$

and

$$
\begin{aligned}
t_{n}^{k}(a, b) \cdot((c \cdot u) \wedge a \wedge b) & \leq t_{n}^{k}(a, b) \cdot(u \wedge a \wedge b) \\
& \leq w \wedge a \wedge b \\
& \leq w .
\end{aligned}
$$

Then

$$
\begin{aligned}
t_{n}^{2 k}(a, b) \cdot((c \cdot u) \wedge a \wedge b) & \leq t_{n}^{k}(a, b) \cdot(c \wedge a \wedge b) \cdot t_{n}^{k}(a, b) \cdot(u \wedge a \wedge b) \\
& \leq d \cdot w .
\end{aligned}
$$


This implies that $t_{n}^{2 k}(a, b) \cdot((c \cdot u) \wedge a \wedge b) \leq(d \cdot w) \wedge a \wedge b$, which is condition 1$)$. Condition 2) can be showed in an analogous way.

In order to prove 3$)$, note that

$$
\begin{aligned}
t_{n}^{k}(a, b) \cdot((c \cdot u) \vee a \vee b) & \leq t_{n}^{k}(a, b) \cdot(c \vee a \vee b) \\
& \leq d \vee a \vee b
\end{aligned}
$$

and

$$
t_{n}^{k}(a, b) \cdot((c \cdot u) \vee a \vee b) \leq t_{n}^{k}(a, b) \cdot(u \vee a \vee b)
$$

Hence,

$$
\begin{aligned}
t_{n}^{2 k}(a, b) \cdot((c \cdot u) \vee a \vee b) & \leq t_{n}^{k}(a, b) \cdot(c \vee a \vee b) \cdot t_{n}^{k}(a, b) \cdot(u \vee a \vee b) \\
& \leq(d \vee a \vee b) \cdot(w \vee a \vee b) .
\end{aligned}
$$

Straightforward computations show that

$$
(d \vee a \vee b) \cdot(w \vee a \vee b) \leq(d \cdot w) \vee a \vee b
$$

Thus,

$$
t_{n}^{2 k}(a, b)((c \cdot u) \vee a \vee b) \leq(d \cdot w) \vee a \vee b .
$$

So we have obtained condition 3$)$. Condition 4$)$ is similarly proved.

Finally we will prove condition 5). Since $t_{n}^{k}(a, b) \leq c \rightarrow d$ and $t_{n}^{k}(a, b) \leq u \rightarrow w$ then $t_{n}^{2 k}(a, b) \leq(c \rightarrow d) \cdot(u \rightarrow w)$. Besides, it follows from Lemma 3 that $(c \rightarrow$ $d) \cdot(u \rightarrow w) \leq(c \cdot u) \rightarrow(d \cdot w)$, so $t_{n}^{2 k}(a, b) \leq(c \cdot u) \rightarrow(d \cdot w)$. Analogously it can be proved that $t_{n}^{2 k}(a, b) \leq(d \cdot w) \rightarrow(c \cdot u)$. Therefore, $t_{n}^{2 k}(a, b) \leq(c \cdot u) \leftrightarrow(d \cdot w)$.

If $A$ is an algebra, $\theta$ a congruence and $a \in A$, then $a / \theta$ denotes the equivalence class of $a$.

Theorem 10. Let $A \in \mathrm{DLCMI}$ and $a, b \in A$. Then $\theta(a, b)=R(a, b)$.

Proof. Notice that items 1)-5) from Lemma 8 are also true by replacing $n$ by $n+1$. Then it follows from lemmas 6 , 8 and 9 that $R(a, b)$ is a congruence that contains the pair $(a, b)$.

Let $\tau$ be a congruence such that $(a, b) \in \tau$. We will prove that $R(a, b) \subseteq \tau$. Let $(c, d) \in R(a, b)$ and $(n, k)$ a pair of natural numbers associated with $(c, d)$. Since $(a, b) \in \tau$ then $(a \leftrightarrow b, 1) \in \tau$, which implies that $\left(t_{n}^{k}(a, b), 1\right) \in \tau$. Hence, it follows from $(\mathrm{C} 1)$ and $(\mathrm{C} 2)$ that

$$
\begin{aligned}
& (c \wedge a \wedge b) / \tau \leq(d \wedge a \wedge b) / \tau, \\
& (d \wedge a \wedge b) / \tau \leq(c \wedge a \wedge b) / \tau, \\
& (c \vee a \vee b) / \tau \leq(d \vee a \vee b) / \tau, \\
& (d \vee a \vee b) / \tau \leq(c \vee a \vee b) / \tau .
\end{aligned}
$$

Thus, $(c \wedge a \wedge b, d \wedge a \wedge b) \in \tau$ and $(c \vee a \vee b, d \vee a \vee b) \in \tau$. Then, by Lemma 5 we conclude that $(c, d) \in \tau$. Hence, $R(a, b) \subseteq \tau$. Therefore, $\theta(a, b)=R(a, b)$.

The theorem shows that DLCMI has locally equationally definable principal congruences by the family of quaternary terms QT introduced immediately after Re$\operatorname{mark}[1]$

The following result, which is [29, Theorem 2.2], follows from Theorem 10] It shows that $\mathrm{WH}$ has locally equationally definable principal congruences.

Corollary 11. Let $A \in \mathrm{WH}$ and $a, b \in A$. Then $(c, d) \in \theta(a, b)$ if and only if there exists a natural number $n$ that satisfies the following conditions:

a) $c \wedge a \wedge b \wedge t_{n}(a, b)=d \wedge a \wedge b \wedge t_{n}(a, b)$,

b) $(c \vee a \vee b) \wedge t_{n}(a, b)=(d \vee a \vee b) \wedge t_{n}(a, b)$,

c) $t_{n}(a, b) \leq c \leftrightarrow d$ 
The next corollary characterizes the principal congruences of the algebras of IDCRL using Theorem 10.

Corollary 12. Let $A \in \mathrm{IDCRL}$ and $a, b \in A$. Then $(c, d) \in \theta(a, b)$ if and only if there exists a natural number $k$ such that $(a \leftrightarrow b)^{k} \leq c \leftrightarrow d$.

Proof. Let $A \in \mathrm{IDCRL}, a, b \in A$ and $n, k$ natural numbers. Since $1 \rightarrow(a \leftrightarrow b)=$ $a \leftrightarrow b$ then $t_{n}(a, b)=a \leftrightarrow b$, so $t_{n}(a, b)^{k}=(a \leftrightarrow b)^{k}$. In what follows we will show that condition (C3) implies conditions (C1) and (C2).

Assume that there exists a natural number $k$ such that

$$
(a \leftrightarrow b)^{k} \leq c \leftrightarrow d .
$$

Since $(a \leftrightarrow b)^{k} \leq c \rightarrow d$, then $c \cdot(a \leftrightarrow b)^{k} \leq d$. Taking into account that $c \wedge a \wedge b \leq c$ we obtain that

$$
\begin{aligned}
(c \wedge a \wedge b) \cdot(a \leftrightarrow b)^{k} & \leq c \cdot(a \leftrightarrow b)^{k} \\
& \leq d .
\end{aligned}
$$

Then,

$$
(c \wedge a \wedge b) \cdot(a \leftrightarrow b)^{k} \leq d,
$$

which implies that $(c \wedge a \wedge b) \cdot(a \leftrightarrow b)^{k} \leq d \wedge a \wedge b$. Analogously it can be showed that $(d \wedge a \wedge b) \cdot(a \leftrightarrow b)^{k} \leq c \wedge a \wedge b$. In order to prove that $(c \vee a \vee b) \cdot(a \leftrightarrow b)^{k} \leq d \vee a \vee b$ first note the equality

$$
(c \vee a \vee b) \cdot(a \leftrightarrow b)^{k}=\left(c \cdot(a \leftrightarrow b)^{k}\right) \vee\left((a \vee b) \cdot(a \leftrightarrow b)^{k}\right)
$$

Since $c \cdot(a \leftrightarrow b)^{k} \leq d$ and $(a \vee b) \cdot(a \leftrightarrow b)^{k} \leq a \vee b$ then

$$
(c \vee a \vee b) \cdot(a \leftrightarrow b)^{k} \leq d \vee a \vee b .
$$

Similarly, it can be showed that $(d \vee a \vee b) \cdot(a \leftrightarrow b)^{k} \leq c \vee a \vee b$.

Hence, we have proved that condition (C3) implies conditions (C1) and (C2). The rest of the proof follows from Theorem 10.

Corollary 12 can also be deduced from results due to Agliano; more precisely in [1] Agliano described the principal congruences of BCI-monoids. It is part of the folklore that if $(A, \wedge, \vee, \cdot, \rightarrow, 1)$ is an integral commutative residuated lattice then the congruences of $(A, \wedge, \vee, \cdot, \rightarrow, 1)$ coincide with the congruences of $(A, \wedge, \cdot, \rightarrow, 1)$, which is the underlying BCI-monoid of $(A, \wedge, \vee, \cdot, \rightarrow, 1)$. Hence, it follows from [1, pp. 409] that if $\theta$ is a congruence of $(A, \wedge, \vee, \cdot, \rightarrow, 1)$ and $a, b \in A$, then $(c, d) \in \theta(a, b)$ if and only if there is a natural number $k$ such that $(a \leftrightarrow b)^{k} \leq c \leftrightarrow d$. Corollary 12 is a particular case of the above mentioned property, when the underlying lattice of $(A, \wedge, \vee, \cdot, \rightarrow, 1)$ is distributive.

\section{Compatible functions}

Let $A \in$ DLCMI. In this section we give a necessary and sufficient condition for a function $f: A^{n} \rightarrow A$ to be compatible. We also find conditions on a binary function $g: A \times A \rightarrow A$ that imply that the function $a \mapsto \min \{b \in A: g(a, b) \leq$ $b\}$ is compatible when defined. We will employ similar ideas to those used in [10, 17, 27, 28, 29].

Definition 5. Let $A$ be an algebra and let $f: A^{n} \rightarrow A$ a function.

1. We say that $f$ is compatible with a congruence $\theta$ of $A$ if $\left(a_{i}, b_{i}\right) \in \theta$ for $i=$ $1, \ldots, n$ implies $\left(f\left(a_{1}, \ldots, a_{n}\right), f\left(b_{1}, \ldots, b_{n}\right)\right) \in \theta$.

2. We say that $f$ is a compatible function of $A$ provided it is compatible with all the congruences of $A$. 
Let $A$ be an algebra and $f: A^{n} \rightarrow A$ a function. Then $f$ is compatible if and only if the algebras $A$ and $\langle A, f\rangle$ have the same congruences. For $n=1, f$ is compatible if and only if $(f(a), f(b)) \in \theta(a, b)$ for every $a, b \in A$. The simplest examples of compatible functions on an algebra are the polynomial functions; note that in particular, all term functions (and constant functions) are compatible [24].

Proposition 1. Let $A \in \mathrm{DLCMI}$ and $f: A \rightarrow A$ a function. The following conditions are equivalent:

1) $f$ is compatible.

2) For every $a, b \in A$ there are natural numbers $n$ and $k$ that satisfy the following conditions:

(Cf1) $t_{n}^{k}(a, b) \cdot(f(a) \wedge a \wedge b) \leq f(b) \wedge a \wedge b$,

(Cf2) $t_{n}^{k}(a, b) \cdot(f(a) \vee a \vee b) \leq f(b) \vee a \vee b$,

(Cf3) $t_{n}^{k}(a, b) \leq f(a) \leftrightarrow f(b)$.

Proof. It follows from Theorem 10 and Remark 2

Remark 3. Note that condition (Cf3) of Proposition 1 can be replaced by $t_{n}^{k}(a, b) \leq$ $f(a) \rightarrow f(b)$.

Let $A$ be an algebra, $f: A^{n} \rightarrow A$ a function and $\vec{a}=\left(a_{1}, \ldots, a_{n}\right) \in A^{n}$. For $i=1, \ldots, n$, define the unary functions $f_{i}^{\vec{a}}: A \rightarrow A$ by

$$
f_{i}^{\vec{a}}(x)=f\left(a_{1}, \ldots, a_{i-1}, x, a_{i+1}, \ldots, a_{n}\right) .
$$

Then, we have the following characterization for the compatibility of an $n$-ary function: $f$ is compatible if and only if for every $\vec{a} \in A^{n}$ and every $i=1, \ldots, n$, the functions $f_{i}^{\vec{a}}: A \rightarrow A$ are compatible. Hence, Proposition 1 allows us to characterize compatible $n$-ary functions in the variety DLCMI.

Corollary 13. Let $A \in \mathrm{WH}$ and $f: A \rightarrow A$ a function. Then $f$ is compatible if and only if for every $a, b \in A$ there exists $n \in \mathbb{N}$ that satisfies the following conditions:

a) $f(a) \wedge a \wedge b \wedge t_{n}(a, b)=f(b) \wedge a \wedge b \wedge t_{n}(a, b)$,

b) $(f(a) \vee a \vee b) \wedge t_{n}(a, b)=(f(b) \vee a \vee b) \wedge t_{n}(a, b)$,

c) $t_{n}(a, b) \leq f(a) \leftrightarrow f(b)$.

Proof. It follows from Proposition 1

Corollary 13 was also proved in [29, Corollary 3.2].

Corollary 14. Let $A \in$ IDCRL and $f: A \rightarrow A$ a function. Then $f$ is compatible if and only if for every $a, b \in A$ there is a natural number $k$ such that $(a \leftrightarrow b)^{k} \leq$ $f(a) \leftrightarrow f(b)$.

Proof. It follows from Corollary 12

The characterization of unary compatible functions for algebras in IDCRL given in Corollary 14 is exactly the characterization of unary compatible functions given by Agliano in [1, pp. 410] for BCI-monoids. Thus, the description of unary compatible functions in IDCRL is also a direct consequence from [1.

Independently from [1, Castiglioni, Menni and Sagastume presented in 10, Theorem 8] a description of the compatible functions in commutative residuated lattices. The unary case of [10, Theorem 8] for the case of integral commutative residuated lattices whose underlying lattice is distributive says that if $A \in$ IDCRL and $f: A \rightarrow A$ is a function, then $f$ is compatible if and only if for every $a, b \in A$ there is a natural number $k$ such that $s(a, b)^{k} \leq s(f(a), f(b))$, where $s(a, b)=(a \rightarrow b) \cdot(b \rightarrow a)$. The proof of the above mentioned property can be easily adapted in order to obtain Corollary 14 
Let $A \in \mathrm{DLCMI}$; if $g$ is a binary function $g$ on $A$, we want to find conditions implying that the function $a \mapsto \min \{b \in A: g(a, b) \leq b\}$ is compatible whenever it is defined.

Definition 6. Let $A$ be a poset and let $g: A \times A \rightarrow A$ be a function. We say that $g$ satisfies condition $(\mathbf{M})$ if the following condition holds:

For all $a, b, c \in A, c \geq b$ implies $g(a, c) \leq g(a, b)$.

If $A$ is a $\vee$-semilattice and $g$ is a function that satisfies condition $(\mathbf{M})$, then $g(a, g(a, b) \vee b) \leq g(a, b) \vee b$ for every $a, b \in A$.

Lemma 15. Let $A$ be $a \vee$-semilattice, and let $g: A \times A \rightarrow A$ be a function that satisfies condition $(\mathbf{M})$. The following conditions are equivalent:

(a) There is a map $f: A \rightarrow A$ given by $f(a)=\min \{b \in A: g(a, b) \leq b\}$.

(b) There exists a map $h: A \rightarrow A$ that satisfies the following conditions for every $a, b \in A$ :

(i) $g(a, h(a)) \leq h(a)$,

(ii) $h(a) \leq g(a, b) \vee b$.

Moreover, in this case we have that $f=h$.

Proof. It follows from [10, Lemma 15].

Let $A$ be an algebra and $g: A \times A \rightarrow A$ a function. For every $a \in A$ define the function $g_{a}: A \rightarrow A$ by $g_{a}(a, b)=g(a, b)$. We say that $g$ is compatible in the first variable if $g_{a}$ is compatible for every $a \in A$.

We apply Lemma 15 in order to prove the following proposition.

Proposition 2. Let $A \in \mathrm{DLCMI}$ and let $f: A \rightarrow A$ be a function. The following conditions are equivalent:

1. $f$ is compatible.

2. There exists a function $g: A \times A \rightarrow A$ that satisfies $(\mathbf{M})$, compatible in the first variable and such that $f(a)=\min \{b \in A: g(a, b) \leq b\}$.

3. There exists a function $\hat{g}: A \times A \rightarrow A$ that satisfies (M), compatible in the first variable and such that satisfies the following conditions for every $a, b \in A$ :

(i) $\hat{g}(a, f(a)) \leq f(a)$,

(ii) $f(a) \leq \hat{g}(a, b) \vee b$.

Moreover, in this case we have that $g=\hat{g}$.

Proof. Assume condition 1., i.e., that $f$ is compatible. We define $g: A \times A \rightarrow A$ by $g(a, b)=f(a)$. Hence, condition 2. is obtained. The equivalence between 2 . and 3 . follows from Lemma 15 .

In order to show that condition 3. implies condition 1., let $a, b \in A$. Since $g$ is compatible in the first variable then it follows from Proposition 1 that there are natural numbers $n$ and $k$ such that

$$
\begin{gathered}
t_{n}^{k}(a, b) \cdot(g(a, f(b)) \wedge a \wedge b) \leq g(b, f(b)) \wedge a \wedge b, \\
t_{n}^{k}(a, b) \cdot(g(a, f(b)) \vee a \vee b) \leq g(b, f(b)) \vee a \vee b, \\
t_{n}^{k}(a, b) \leq g(a, f(b)) \rightarrow g(b, f(b)) .
\end{gathered}
$$

Taking into account (8) and Lemma 15 we have that

$$
\begin{aligned}
t_{n}^{k}(a, b) \cdot(f(a) \wedge a \wedge b) & \leq t_{n}^{k}(a, b) \cdot((g(a, f(b)) \vee f(b)) \wedge a \wedge b) \\
& =t_{n}^{k}(a, b) \cdot((g(a, f(b)) \wedge a \wedge b) \vee(f(b) \wedge a \wedge b)) \\
& =\left(t_{n}^{k}(a, b) \cdot(g(a, f(b)) \wedge a \wedge b)\right) \vee\left(t_{n}^{k}(a, b) \cdot(f(b) \wedge a \wedge b)\right) \\
& \leq(g(b, f(b)) \wedge a \wedge b) \vee(f(b) \wedge a \wedge b) \\
\leq & (f(b) \wedge a \wedge b) \vee(f(b) \wedge a \wedge b) \\
& =f(b) \wedge a \wedge b .
\end{aligned}
$$


Hence,

$$
t_{n}^{k}(a, b) \cdot(f(a) \wedge a \wedge b) \leq f(b) \wedge a \wedge b .
$$

Besides, it follows from (9) and Lemma 15 that

$$
\begin{aligned}
t_{n}^{k}(a, b) \cdot(f(a) \vee a \vee b) & \leq t_{n}^{k}(a, b) \cdot(g(a, f(b)) \vee f(b) \vee a \vee b) \\
& =\left(t_{n}^{k}(a, b) \cdot(g(a, f(b)) \vee a \vee b) \vee\left(t_{n}^{k}(a, b) \cdot(f(b) \vee a \vee b)\right)\right. \\
& \leq(g(b, f(b)) \vee a \vee b) \vee(f(b) \vee a \vee b) \\
& \leq(f(b) \vee a \vee b) \vee(f(b) \vee a \vee b) \\
& =f(b) \vee a \vee b .
\end{aligned}
$$

Thus,

$$
t_{n}^{k}(a, b) \cdot(f(a) \vee a \vee b) \leq f(b) \vee a \vee b .
$$

Finally we will prove that $t_{n}^{k}(a, b) \leq f(a) \rightarrow f(b)$. It follows from (10) and the inequality $g(b, f(b)) \leq f(b)$ that

$$
t_{n}^{k}(a, b) \leq g(a, f(b)) \rightarrow b .
$$

Since $f(a) \leq g(a, f(b)) \vee f(b)$ then $f(a) \rightarrow f(b) \geq(g(a, f(b)) \vee f(b)) \rightarrow f(b)$. But $(g(a, f(b)) \vee f(b)) \rightarrow f(b)=g(a, f(b)) \rightarrow f(b)$, so it follows from (13) that

$$
t_{n}^{k}(a, b) \leq f(a) \rightarrow f(b) .
$$

Therefore, it follows from (11), (12), (14) and Proposition 1 that $f$ is a compatible function.

In the rest of this section we apply Proposition 2 in order to study possible generalizations of the gamma function [7. Example 5.1], the successor function [7, Example 5.2] and the Gabbay's function [7, Example 5.3] considered by Caicedo and Cignoli in 7 as examples of implicit compatible operations on Heyting algebras. These functions were also generalized in different frameworks, as for instance in residuated lattices [10, 12, and in weak Heyting algebras [15, 29.

We start with the following definition that can be found in 9 .

Definition 7. Let $\mathrm{V}$ be a variety of algebras of type $F$ and let $\epsilon(C)$ be a set of identities of type $F \cup C$, where $C$ is a family of new function symbols. We say that $\epsilon(C)$ defines implicitly $C$, if in each algebra $A \in V$ there is at most one family $\left\{f_{A}: A^{n} \rightarrow A\right\}_{f \in C}$ such that $\left(A, f_{A}\right)_{f \in C}$ satisfies the universal closure of the equations in $\epsilon(C)$. In this case we say that each $f$ is implicitly defined in $\mathrm{V}$.

In what follows we will consider $A \in \mathrm{DLCMI}$ and $n$ a natural number.

Example 2. Suppose that the underlying lattice of $A$ is bounded, and write 0 for the smallest element. We define the unary compatible function $\gamma_{n}$ by

$$
\gamma_{n}(a)=\min \left\{b \in A: a \vee \neg b^{n} \leq b\right\},
$$

where $\neg x$ is defined by $x \rightarrow 0$. Equivalently, $\gamma_{n}$ can be implicitly defined by the inequalities

(g1) $a \vee \neg\left(\gamma_{n}(a)\right)^{n} \leq \gamma_{n}(a)$,

(g2) $\gamma_{n}(a) \leq a \vee \neg b^{n} \vee b$.

The function $\gamma_{n}$ preserves the order, i.e., if $a \leq b$, then $\gamma_{n}(a) \leq \gamma_{n}(b)$. In order to show it, let $a \leq b$. By (g2) we have that $\gamma_{n}(a) \leq a \vee \neg\left(\gamma_{n}(b)\right)^{n} \vee \gamma_{n}(b)$. Since $a \leq b$ then $\gamma_{n}(a) \leq b \vee \neg\left(\gamma_{n}(b)\right)^{n} \vee \gamma_{n}(b)$. Besides, by (g1) we obtain $b \vee \neg\left(\gamma_{n}(b)\right)^{n} \leq \gamma_{n}(b)$. Hence, $\gamma_{n}(a) \leq \gamma_{n}(b)$.

Lemma 16. The function $\gamma_{n}$ is characterized as the unary function that satisfies the following conditions for every $a, b$ :

$$
\text { (g3) } \neg\left(\gamma_{n}(0)\right)^{n} \leq \gamma_{n}(0) \text {, }
$$


(g4) $\gamma_{n}(0) \leq b \vee \neg b^{n}$,

(g5) $\gamma_{n}(a)=a \vee \gamma_{n}(0)$.

In particular, $\gamma_{n}$ is a polynomial function on $A$.

Proof. Assume that $\gamma_{n}$ is an unary function that satisfies (g1) and (g2). If we put $a=0$ in (g1) and (g2) then the equations (g3) and (g4) follow. In what follows we will prove (g5). By (g2) with $b=\gamma_{n}(0)$ and by (g3) we obtain $\gamma_{n}(a) \leq a \vee \gamma_{n}(0)$. In order to prove the other inequality, note that it follows from (g1) that $a \leq \gamma_{n}(a)$. Since $\gamma_{n}$ preserves the order then $\gamma_{n}(0) \leq \gamma_{n}(a)$. Thus, $a \vee \gamma_{n}(0) \leq \gamma_{n}(a)$. Hence, $\gamma_{n}(a)=a \vee \gamma_{n}(0)$, which is (g5). Conversely, assume that $\gamma_{n}$ is a unary function that satisfies (g3), (g4) and (g5). Condition (g2) follows from (g4) and (g5). Finally we will prove (g1). Since (g5) holds, then $\gamma_{n}(0) \leq \gamma_{n}(a)$, so $\left(\gamma_{n}(0)\right)^{n} \leq\left(\gamma_{n}(a)\right)^{n}$. Taking into account (g3) we obtain $\neg\left(\gamma_{n}(a)\right)^{n} \leq \neg\left(\gamma_{n}(0)\right)^{n} \leq \gamma_{n}(0)$. Thus, $a \vee$ $\neg\left(\gamma_{n}(a)^{n}\right) \leq a \vee \gamma_{n}(0)=\gamma_{n}(a)$. Therefore we have showed condition (g1), which was our aim.

It follows from Lemma 16 that $\gamma_{n}$ is a polynomial function, which implies that $\gamma_{n}$ is a compatible function. Then we have obtained an alternative proof for the compatibility of $\gamma_{n}$.

Remark 4. Let us write $\gamma$ for the function on Heyting algebras given in 7, Example 5.1]. It was proved in [11 that $\gamma$ can be defined by $\gamma_{n}(a)=\min \{b \in A: a \vee \neg b \leq b\}$, or equivalently, as the unary function that satisfies the conditions (g3), (g4) and (g5) from Lemma 16. Thus, on Heyting algebras we have that the definitions of $\gamma$ and $\gamma_{1}$ are the same.

Example 3. We define the unary compatible function $S_{n}$ by

$$
S_{n}(a)=\min \left\{b \in A: b^{n} \rightarrow a \leq b\right\} .
$$

Equivalently, $S_{n}$ can be implicitly defined by the inequalities

(S1) $\left(S_{n}(a)\right)^{n} \rightarrow a \leq S_{n}(a)$

(S2) $S_{n}(a) \leq b \vee\left(b^{n} \rightarrow a\right)$.

On Heyting algebras the function $S_{1}$ is the successor function. For details about the successor function on Heyting algebras see [18, 25, 26.

Example 4. Assume that the underlying lattice of $A$ is bounded. Define the unary compatible function $G_{n}$ by

$$
G_{n}(a)=\min \left\{b \in A:\left(b^{n} \rightarrow a\right) \wedge \neg \neg a \leq b\right\} .
$$

In an equivalent way, $G_{n}$ can be implicitly defined by the inequalities

(G1) $\left(\left(G_{n}(a)\right)^{n} \rightarrow a\right) \wedge \neg \neg a \leq G_{n}(a)$,

(G2) $G_{n}(a) \leq b \vee\left(\left(b^{n} \rightarrow a\right) \wedge \neg \neg a\right)$.

On Heyting algebras the function $G_{1}$ is the Gabbay's function, which will be denoted $G$. The description of $G$ as the minimum of certain set was proved in [11. See also 22 for historical remarks about $G$.

\section{Other CONNECTIONS With EXISTing Literature}

In 13. Celani introduced distributive lattices with fusion and implication. An algebra $(A, \wedge, \vee, \rightarrow, 0,1)$ of type $(2,2,2,0,0)$ is a distributive lattice with implication if $(A, \wedge, \vee, 0,1)$ is a bounded distributive lattice and for every $a, b, c \in A$ the following conditions are satisfied:

(I1) $(a \rightarrow b) \wedge(a \rightarrow c)=a \rightarrow(b \wedge c)$,

(I2) $(a \rightarrow c) \wedge(b \rightarrow c)=(a \vee b) \rightarrow c$,

(I3) $0 \rightarrow a=a \rightarrow 1=1$. 
An algebra $(A, \wedge, \vee, \cdot, 0,1)$ of type $(2,2,2,0,0)$ is a distributive lattice with fusion if $(A, \wedge, \vee, 0,1)$ is a bounded distributive lattice and for every $a, b, c \in A$ the following conditions are satisfied:

(F1) $a \cdot(b \vee c)=(a \cdot b) \vee(a \cdot c)$,

$(\mathrm{F} 2)(b \vee c) \cdot a=(b \cdot c) \vee(a \cdot c)$,

(F3) $0 \cdot a=a \cdot 0=0$.

An algebra $(A, \wedge, \vee, \cdot, \rightarrow, 0,1)$ is a bounded distributive lattice with fusion and implication if $(A, \wedge, \vee, \rightarrow, 0,1)$ is a bounded distributive lattice with implication and $(A, \wedge, \vee, \cdot, 0,1)$ is a bounded distributive lattice with fusion. Straightforward computations show that for every $(A, \wedge, \vee, \cdot, \rightarrow, 1) \in \mathrm{DLCMI}$ with a smallest element 0 we have that the algebra $(A, \wedge, \vee, \cdot, \rightarrow, 0,1)$ is a distributive lattice with fusion and implication.

There are other connections of the present paper with existing literature. A generalized commutative residuated lattice [28, Def. 1.1] is an algebra $(A, \wedge, \vee, \cdot, \rightarrow, e)$ of type $(2,2,2,2,0)$ that satisfies the following conditions: $(A, \cdot, e)$ is a commutative monoid, $(A, \vee, \wedge)$ is a lattice, and for every $a, b, c \in A$ the following properties hold: $a \rightarrow(b \wedge c)=(a \rightarrow b) \wedge(a \rightarrow c),(a \vee b) \rightarrow c=(a \rightarrow c) \wedge(b \rightarrow c)$, $(a \rightarrow b) \cdot(b \rightarrow c) \leq a \rightarrow c$, and $e \leq a \rightarrow a$. We write GCRL for the variety of generalized commutative residuated lattices. Clearly, DLCMI is a subvariety of GCRL.

Remark 5. Let $A \in \mathrm{GCRL}, a \in A$ and $n \geq 1$. We define $\square(a):=e \rightarrow a$. As usual, also define $\square^{2}(a)=\square(\square(a))$ and $a^{2}=a \cdot a$. Notice that if $A \in$ DLCMI, the definition of $\square$ given before collapses to the definition of $\square$ given in Section 2 for algebras of DLCMI.

Definition 8. 28, Def. 1.2] A commutative weak residuated lattice is a generalized commutative residuated lattice $(A, \wedge, \vee, \cdot, \rightarrow, e)$ that satisfies the following conditions for every $a, b, c \in A$ :

(R1) $a \cdot(a \rightarrow b) \leq b$,

(R2) $(a \cdot b) \vee(a \cdot c)=a \cdot(b \vee c)$,

(R3) $\square(a) \leq b \rightarrow(b \cdot a)$,

(R4) $a \leq(a \rightarrow e) \rightarrow e$,

(R5) $\square\left(a^{2}\right) \leq \square(a) \cdot \square(a)$,

(R6) $\square(a) \rightarrow(\square(a) \rightarrow e) \leq \square^{2}(a) \rightarrow e$,

(R7) $(\square(a) \rightarrow e) \cdot(\square(a) \rightarrow e) \leq \square\left(a^{2}\right) \rightarrow e$.

We write CWRL for the variety of commutative weak residuated lattices. Commutative weak residuated lattices are a common abstraction of commutative residuated lattices 23 and weak Heyting algebras that satisfy $a \wedge(a \rightarrow b) \leq b$ for every $a, b$. The varieties DLCMI and CWRL are incomparable. In order to show it, first note that there are commutative residuated lattices without largest element (or with underlying lattice not necessarily distributive), so CWRL $\nsubseteq$ DLCMI. Besides, the algebras of CWRL satisfy the inequality $a \cdot(a \rightarrow b) \leq b$ for every $a, b$. Since the previous inequality is not satisfied by the algebra given in Example 1, then DLCMI $\nsubseteq$ CWRL.

Acknowledgments. This project has received funding from the European Union's Horizon 2020 research and innovation programme under the Marie SklodowskaCurie grant agreement No. 689176.

The first author was also partially supported by the research grant 2014 SGR 788 from the government of Catalonia and by the research projects MTM201125747 and MTM2016-74892-P from the government of Spain, which includes FEDER funds from the European Union and he also acknowledges financial support from the 
Spanish Ministry of Economy and Competitiveness, through the "María de Maeztu" Programme for Units of Excellence in R\&D (MDM-2014-0445). The second author was also supported by CONICET Project PIP 112-201501-00412.

\section{REFERENCES}

[1] Agliano P., Ternary deductive terms in residuated structures. Acta Sci. Math. (Szeged) 68, 397-429 (2002).

[2] Balbes R. and Dwinger P., Distributive Lattices, University of Missouri Press, 1974.

[3] Bezhanishvili N. and Gehrke M., Finitely generated free Heyting algebras via Birkhoff duality and coalgebra. Logical Methods in Computer Science 7, 1-24 (2011).

[4] Blok, W. and D. Pigozzi, Local Deduction Theorems in Algebraic Logic, in Algebraic Logic, edited by H. Andréka, J. D. Monk and I. Németi, Colloq. Math. Soc. János Bolyai 54, 75-109, North-Holland, Amsterdam, 1991.

[5] Blok W.J. and Pigozzi D, Abstract algebraic logics and the deduction theorem, Manuscript, 2001. http://orion.math.iastate.edu/dpigozzi/

[6] Burris H. and Sankappanavar H.P, A Course in Universal Algebra. Springer Verlag, New York, 1981.

[7] Caicedo X. and Cignoli R., An algebraic approach to intuitionistic connectives. Journal of Symbolic Logic 4, 1620-1636 (2001).

[8] Caicedo X., Implicit connectives of algebraizable logics. Studia Logica 78, No. 3, 155-170 (2004).

[9] Caicedo X., Implicit operations in MV-algebras and the connectives of Lukasiewicz logic. Lecture Notes in Computer Science, vol. 4460, no. 1, 50-68 (2007).

[10] Castiglioni J.L., Menni M. and Sagastume M., Compatible operations on commutative residuated lattices. JANCL 18, 413-425 (2008).

[11] Castiglioni J.L., Sagastume M. and San Martín H.J., On frontal Heyting algebras. Reports on Mathematical Logic, vol. 45, 201-224 (2010).

[12] Castiglioni J.L. and San Martín H.J., Compatible operations on residuated lattices. Studia Logica 98 (1-2), 203-222 (2011).

[13] Celani S.A, Distributive Lattices with Fusion and Implication. Southeast Asian Bulletin of Mathematics 28, 999-1010 (2004).

[14] Celani S.A. and Jansana R. Bounded distributive lattices with strict implication. Mathematical Logic Quarterly 51, 219-246 (2005).

[15] Celani S.A. and San Martín H.J., Frontal operators in weak Heyting algebras. Studia Logica 100, 91-114 (2012).

[16] Cignoli R., D'Ottaviano I. and Mundici D., Algebraic Foundations of Many-Valued Reasoning. Trends in Logic, Studia Logica Library, Vol. 7, Kluwer Academic Publishers (2000).

[17] Ertola R. and San Martín H.J., On some compatible operations on Heyting algebras. Studia Logica 98, 331-345 (2011).

[18] Esakia L., The modalized Heyting calculus: a conservative modal extension of the Intuitionistic Logic. Journal of Applied Non-Classical Logics 16, 349-366 (2006).

[19] Font J.M., Abstract Algebraic logic. An Introductory Course, College Publications, London, 2016.

[20] Fried E., Grätzer G. and Quackenbush R. W., The equational class generated by weakly associative lattices with the unique bound property. Ann. Univ. Sci. Budapest. Eštvšs Sect. Math., 205-211 (1979/80).

[21] Fried E., Grätzer G. and Quackenbush R. W., Uniform congruence schemes. Algebra Universalis $10,176-188(1980)$.

[22] Gabbay D.M., On some new intuitionistic propositional connectives. Studia Logica 36, 127139 (1977)

[23] Hart J., Raftery L. and Tsinakis C., The structure of commutative residuated lattices. Internat. J. Algebra Comput. 12, 509-524 (2002).

[24] Kaarli K. and Pixley A.F., Polynomial completeness in algebraic systems, Chapman and Hall/CRC, 2001.

[25] Kuznetsov A. V. On the Propositional Calculus of Intuitionistic Provability. Soviet Math. Dokl. 32, 18-21 (1985).

[26] Muravitsky A.Y., Logic KM: A biography, in: Leo Esakia on Duality of Modal and Intuitionistic logics. Series: Outstanding Contributions to Logic, 4, Springer (2014), 147-177.

[27] San Martín H.J., Compatible operations in some subvarieties of the variety of weak Heyting algebras, 8th Conference of the European Society for Fuzzy Logic and Technology (EUSFLAT 2013), $475-480$ 
[28] San Martín H.J., Compatible operations on commutative weak residuated lattices. Algebra Universalis, vol. 73, no. 2, 143-155 (2015).

[29] San Martín H.J., Principal congruences in weak Heyting algebras. Algebra Universalis, vol. 75, no. 4, 405-418 (2016). 
Ramon Jansana,

Barcelona Graduate School of Mathematics

Philosophy Department Universitat de Barcelona.

Montalegre, 6,

08001, Barcelona,

España.

jansana@ub.edu

Hernán Javier San Martín,

Departamento de Matemática,

Facultad de Ciencias Exactas (UNLP),

and CONICET.

Casilla de correos 172,

La Plata (1900),

Argentina.

hsanmartin@mate.unlp.edu.ar 